\title{
The effects of short term lipid infusion on plasma and hepatic bile lipids in humans
}

\author{
R Pakula, F M Konikoff, A M Moser, F Greif, A Tietz, T Gilat, M Rubin
}

\begin{abstract}
Background-Patients on parenteral nutrition have an increased incidence of gall bladder sludge and gallstone disease, thought to be related to bile stasis. Intravenous lipid emulsions, especially those containing medium chain triglycerides, have also been shown to have a lithogenic effect on the composition of bile in the gall bladder.
\end{abstract}

Aims-To determine whether lipid infusion influences hepatic bile composition in patients with an indwelling $T$ tube following cholecystectomy and choledochotomy.

Methods-In eight patients undergoing the above surgical procedure, the time at which effects of the interrupted enterohepatic circulation were minimal was determined. Twenty two cholesterol gallstone patients with bile fistula were then randomised to receive an infusion of a lipid emulsion containing either long chain triglycerides or a mixture of long and medium chain triglycerides.

Results-Lipid infusion resulted in a significant increase in plasma levels of triglycerides and phospholipids. Both lipid emulsions caused an increase in hepatic biliary cholesterol level and cholesterol saturation index, but this effect was more pronounced with medium chain triglycerides. The fatty acid composition of biliary phospholipids showed a significant enrichment of linoleic acid by both lipid infusions.

Conclusions-Infusion of triglycerides causes lithogenic changes in hepatic bile composition in humans, the lithogenic effect of infusion of medium chain triglycerides being more pronounced than that of long chain triglycerides. This effect, coupled with gall bladder stasis, may be responsible for the increased risk of biliary sludge and gallstone formation in patients on long term lipid infusion.

(Gut 1999;45:453-458)

Keywords: lipid emulsion; long chain triglycerides; medium chain triglycerides; bile; cholesterol; gallstones

Several reports have shown an increased incidence of gall bladder disease in patients on long term parenteral nutrition. ${ }^{12}$ In addition to biliary sludge, some patients develop gallstones and progress to symptomatic biliary disease. The most common explanation for these effects is bile stasis caused by failure of gall bladder emptying, as a result of reduced secre- tion of intestinal hormones which are normally released after oral food intake. ${ }^{34}$ This is supported by the finding that administration of cholecystokinin, which induces gall bladder contraction, can prevent the consequent complications. $^{56}$

However, studies in rats $^{78}$ and patients with non-cholesterol gallstones, ${ }^{9}$ have shown that intravenous lipid infusion also increases bile lithogenicity by altering bile composition. More specifically, infusion of lipid emulsions containing medium chain triglycerides (MCT) had more pronounced effects on gall bladder bile composition than emulsions containing long chain triglycerides (LCT) in noncholesterol gallstone patients. ${ }^{9}$ Furthermore, administration of LCT to rats caused an increase in bile lithogenicity when given orally or intravenously. ${ }^{8}$ A recent study in prairie $\operatorname{dogs}^{10}$ showed that diets supplemented with MCT induced a significant increase in biliary cholesterol levels. These lithogenic effects may contribute significantly to the development of cholesterol gallstones, particularly in patients on long term infusion of lipid emulsions. As the composition of gall bladder bile is determined by the hepatic bile as well as by absorptive processes within the gall bladder, current data do not allow one to determine whether the lithogenic effect of lipid infusion stems from a direct effect on hepatic bile composition or an indirect effect on gall bladder bile. We postulate that intravenous lipid infusion can affect hepatic bile composition.

Human hepatic bile can be obtained for metabolic studies from patients following cholecystectomy and choledochotomy who are left for medical reasons with a temporary indwelling biliary drainage in the form of a $\mathrm{T}$ tube. Biliary drainage, however, interrupts the enterohepatic circulation, and can result in bile acid deficiency. It disrupts the intestinal absorption of cholesterol, ${ }^{11}{ }^{12}$ and increases the conversion of cholesterol to bile acids in the liver. ${ }^{13-15}$ Clamping of the $\mathrm{T}$ tube prior to its removal restores bile flow and the enterohepatic circulation, enabling the metabolic changes to revert to the preoperative state. The exact timeframe of these events is, however, controversial. Supersaturated bile, for example, becomes unsaturated with cholesterol within three days after $\mathrm{T}$ tube clamping, and the biliary lipid composition is believed to stabilise within a week. ${ }^{16}$ In order to use $\mathrm{T}$ tube bile to study the effects of lipid infusion on hepatic

Abbreviations used in this paper: $\mathrm{BS}$, bile salts; CSI, cholesterol saturation index; LCT, long chain triglycerides; MCT, medium chain triglycerides; PL, phospholipids; TG, triglycerides.
Accepted for publication 18 March 1999 
Table 1 Patient characteristics

\begin{tabular}{|c|c|c|c|c|c|}
\hline & \multicolumn{2}{|l|}{ Study I } & \multicolumn{3}{|l|}{ Study II } \\
\hline & $\begin{array}{l}\text { Bile fistula } \\
(n=8)\end{array}$ & $\begin{array}{l}\text { Control } \\
(n=6)\end{array}$ & $\begin{array}{l}L C T \\
(n=10)\end{array}$ & $\begin{array}{l}M C T / L C T \\
(n=12)\end{array}$ & $\begin{array}{l}\text { Control } \\
(n=5)\end{array}$ \\
\hline Withdrawn & 2 & 0 & 2 & 1 & 0 \\
\hline $\operatorname{Sex}(F / M)$ & $8 / 0$ & $4 / 2$ & $8 / 2$ & $11 / 1$ & $5 / 0$ \\
\hline Age $(y)$ & $70(21)$ & $60(15)$ & $62(18)$ & $72(6)$ & $69(4)$ \\
\hline $\mathrm{BMI}\left(\mathrm{kg} / \mathrm{m}^{2}\right)$ & $28(7)$ & $25(4)$ & $29(7)$ & $28(4)$ & $27(3)$ \\
\hline $\begin{array}{l}\text { Stone composition } \\
\quad \text { (cholesterol/pigment) }\end{array}$ & $6 / 2$ & $4 / 2$ & $10 / 0$ & $12 / 0$ & $5 / 0$ \\
\hline
\end{tabular}

Results are expressed as mean (SD). BMI, body mass index; LCT, long chain triglycerides; MCT, medium chain triglycerides. bile composition, knowledge of this timeframe is pertinent.

The present study was undertaken to determine the effects of a short term infusion of two lipid emulsions (MCT/LCT and LCT) on lipid composition of plasma and hepatic bile of patients with cholesterol gallstones and a bile fistula. As hepatic bile was to be accessed via a $\mathrm{T}$ tube, we first investigated the sequence of events occurring during recovery of plasma and biliary lipids in these patients following biliary surgery.

\section{Materials and methods SUBJECTS}

This paper will report the results of both phases of the study. Fourteen patients were included in study I. Eight were gallstone patients who had undergone cholecystectomy, choledochotomy, and $\mathrm{T}$ tube placement, based on surgical indications; two were withdrawn due to fever and diarrhoea during the study. In addition, six gallstone patients, who had undergone elective cholecystectomy, without choledochotomy served as controls.

Twenty seven patients with ultrasonography proved gallstones who were scheduled to undergo elective cholecystectomy, choledochotomy, and $\mathrm{T}$ tube placement were enrolled in study II. Results of three patients were excluded due to inadequate biliary lipid concentration. Eleven patients were infused with an MCT/LCT emulsion and eight with an LCT emulsion. Five additional cholesterol gallstone patients who had undergone the same surgical procedure served as controls and were infused with a $5 \%$ glucose- $0.9 \%$ saline solution. All patients had cholesterol gallstones. Table 1 presents the demographic and clinical data of patients in both studies.

All patients, in both study groups, were well nourished and their hepatic, thyroid, and renal functions were within normal limits. Exclusion criteria were: obesity (BMI >2 SD of normal), recent weight loss (more than 10\% during past six months), significant hypercholesterolaemia (cholesterol $>2700 \mathrm{mg} / \mathrm{l}$ ), hypertriglyceridaemia (triglycerides $>2500 \mathrm{mg} / \mathrm{l}$ ), diabetes mellitus, or cholangitis. The study was approved by the local ethics committee, and informed consent was obtained from each participant before enrolment in the study.
STUDY PROTOCOL

Study I: Postoperative stabilisation of plasma and hepatic biliary lipids

The study was initiated on the day when the $\mathrm{T}$ tube was clamped (6 (2) days after the operation). Bile samples were obtained immediately before clamping (C-0), as well as on the second (C-2), third (C-3), fourth (C-4), and sixth day (C-6) after clamping. Plasma samples were obtained before the operation as well as on C-0, C-2, C-4, C-6, and at check up approximately six months postoperatively. Plasma samples from the control patients were collected according to the same schedule, C-0 being the fourth postoperative day. All blood and bile samples were collected at 800 am after an overnight fast. Bile was collected by gravity from the $T$ tube over 30 minutes each time. In between sampling the $T$ tube was kept clamped throughout the study period.

\section{Study II: Effects of lipid infusion}

After 8 (3) days postoperatively, when the T tube had been clamped for about 5 (1) days, patients were randomised to receive an infusion of lipid emulsions in a double blinded manner. After an overnight fast patients were infused for six hours, at a rate of $0.2 \mathrm{~g}$ triglycerides (TG)/ $\mathrm{kg} / \mathrm{h}$ (maximum $16.6 \mathrm{~g} \mathrm{TG} / \mathrm{h}$ ) with either $20 \%$ MCT/LCT emulsion or $20 \%$ LCT emulsion, followed by $2000 \mathrm{ml}$ of glucose-saline solution for 18 hours. During the study period, oral intake was restricted to clear fluids. Blood samples were withdrawn prior to and at the end of the six hour infusion period for determination of plasma lipids and lipoproteins. Hepatic bile samples were collected by gravity from the $\mathrm{T}$ tube for 30 minutes at time 0 and after 6,9 , and 24 hours. The first $5 \mathrm{ml}$ of each bile sample was discarded. In between sampling, the $\mathrm{T}$ tube was kept clamped. The control group of patients was infused with $2500 \mathrm{ml}$ glucosesaline solution for 24 hours; bile samples were collected at the same time points as for the study group.

\section{BILE ANALYSIS}

An aliquot $(1 \mathrm{ml})$ of the collected bile was frozen immediately at $-70^{\circ} \mathrm{C}$ for subsequent lipid analysis, ${ }^{17}$ performed within four weeks. Bile lipids were extracted as described by Folch et al. ${ }^{18}$ Bile salt (BS) concentration was determined enzymatically, ${ }^{19}$ cholesterol by the method of Abbell et al, ${ }^{20}$ and phospholipids (PL) as described by Bartlett. ${ }^{21}$ The cholesterol saturation index (CSI) was calculated using the critical tables of Carey. ${ }^{22}$ The gross appearance and chemical analysis of the stones, as well as the presence or absence of cholesterol monohydrate crystals in the sediment, were used to classify stone composition. ${ }^{23}$

\section{PLASMA LIPID ANALYSIS}

Ten $\mathrm{ml}$ of venous blood was drawn into glass tubes containing EDTA ( $1 \mathrm{mg} / \mathrm{ml}$ blood) and were transferred to the laboratory on iced water for immediate separation by low speed refrigerated centrifugation $\left(1000 \mathrm{~g}, 4^{\circ} \mathrm{C}\right)$ for 10 minutes. Sodium azide ( $1 \mathrm{mg} / \mathrm{ml}$ plasma) was added, and the plasma was then kept at $4^{\circ} \mathrm{C}$ for 


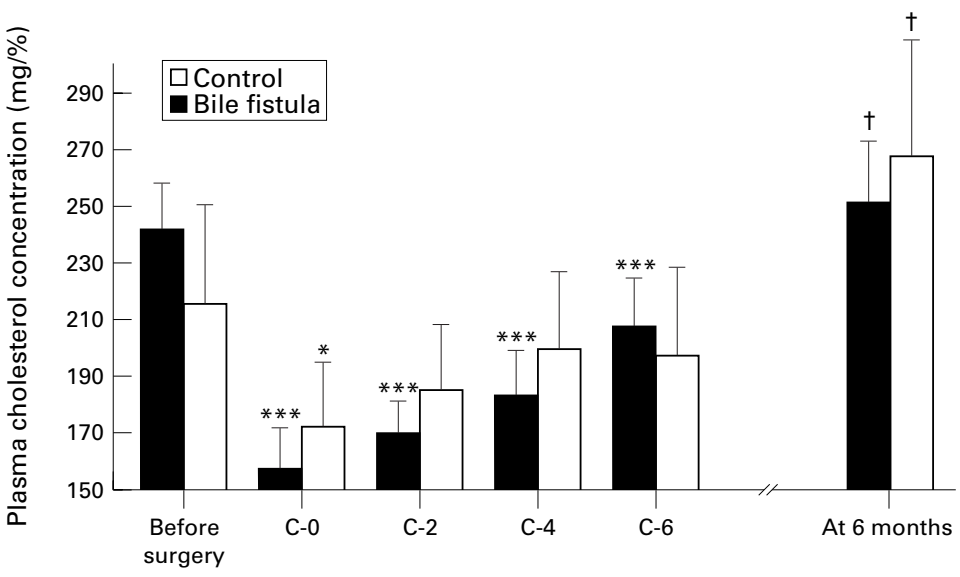

Figure 1 Plasma cholesterol levels during the study period in patients with a bile fistula and controls. From clamping day $(C-0)$ throughout the six days of the study, plasma cholesterol was significantly decreased compared with preoperative levels. ${ }^{\star} p<0.05$ $\star \star \star p<0.001$.

up to five days prior to determination of total plasma lipids. ${ }^{24}$ TG were determined using a commercial kit from Sigma Co. (USA); PL (phosphatidylcholine only), total, and free cholesterol were determined by kits from Boehringer Mannheim GmbH (Germany).

Table 2 Biliary lipid concentrations after T tube clamping

\begin{tabular}{|c|c|c|c|c|c|c|c|c|c|}
\hline & $C-0$ & & $C-2$ & & $C-3$ & & $C-4$ & & $C-6$ \\
\hline Cholesterol (mM) & 2.0 & $(0.9)$ & 2.8 & $(1.2)$ & 3.1 & $(1.3)^{\star}$ & 3.0 & $(1.5)$ & $3.3(1.4)$ \\
\hline Phospholipids (mM) & 3.8 & $(1.6)$ & 8.4 & $(4.1)^{\star}$ & 7.6 & (2.7) & 8.4 & $(2.6)$ & $9.4 \quad(3.4)$ \\
\hline Bile salts (mM) & 9.4 & $(4.1)$ & 27.7 & $(9.8)^{\star \star}$ & 26.0 & (7.7) & 30.7 & $(9.6)$ & $31.9 \quad(9.5)$ \\
\hline Total lipids (g/1) & 8 & (3) & 21 & $(8)^{\star}$ & 20 & (6) & 23 & (7) & 24 \\
\hline \multicolumn{10}{|l|}{ Cholesterol } \\
\hline saturation index & 4.2 & $(2.0)$ & 1.6 & $(0.9)^{\star}$ & 1.8 & $(0.9)$ & 1.4 & $(0.6)$ & $1.4 \quad(0.3)$ \\
\hline Bile flow $(\mathrm{ml} / \mathrm{min})$ & 0.40 & $(0.08)$ & 0.46 & $(0.22)$ & 0.54 & $4(0.14)$ & 0.56 & $5(0.26)$ & $0.36(0.21)$ \\
\hline
\end{tabular}

Results are expressed as mean (SD). Biliary lipid concentrations were measured immediately before clamping $(\mathrm{C}-0)$, as well as on the second $(\mathrm{C}-2)$, third $(\mathrm{C}-3)$, fourth $(\mathrm{C}-4)$, and sixth day (C-6) after clamping.

${ }^{\star} \mathrm{p}<0.05,{ }^{\star \star} \mathrm{p}<0.01$, first significant change in comparison with $\mathrm{C}-0$.

Table 3 Effects of lipid infusion on plasma and lipoprotein lipid concentrations ( $m g / l)$

\begin{tabular}{|c|c|c|c|c|}
\hline & \multicolumn{2}{|l|}{$L C T$} & \multicolumn{2}{|l|}{$M C T / L C T$} \\
\hline & Time 0 & End of infusion & Time 0 & End of infusion \\
\hline Triglycerides & $1310(390)$ & $5690(3060)^{\star \star}$ & $1371(475)$ & $6158(3903)^{\star \star \star}$ \\
\hline $\mathrm{CE}$ & $2070(42.0)$ & $1910(310)$ & 2333 (413) & $2283(335)$ \\
\hline FC & $525(108)$ & $588(84)$ & $551(77)$ & $595(72)$ \\
\hline Phospholipids & $2271(282)$ & $2983(274) \dagger$ & $2322(229)$ & $3171(313) \dagger$ \\
\hline
\end{tabular}

Results are expressed as mean (SD).

${ }^{\star \star} \mathrm{p}<0.01,{ }^{\star \star \star} \mathrm{p}<0.001,+\mathrm{p}<0.005$ versus time 0 .

$\mathrm{CE}$, cholesterol ester; FC, free cholesterol.
ANALYSIS OF BILE PHOSPHOLIPID FATTY ACID COMPOSITION

Aliquots of biliary lipid extracts were saponified with $0.5 \mathrm{M}$ methanolic $\mathrm{KOH}$ for one hour at $50^{\circ} \mathrm{C}^{25}$ and fatty acids were recovered after acidification by hexane extraction. The fatty acids were methylated with diazomethane according to Schlenk and Gellerman. ${ }^{26}$ Methylesters were separated by gas liquid chromatography on a 30m PAG (poly alkylene glycol) column (Supelco, Bellefonte, Pennsylvania, USA; $0.25 \mu \mathrm{m}$ film thickness) at a temperature range of $185-220^{\circ} \mathrm{C}$ using a Hewlett-Packard 5790A gas chromatograph equipped with a flame ionisation detector. The relative composition of a fatty acid mixture was calculated by a Hewlett-Packard 3390A integrator. For quantitative analysis, heptadecanoic acid was added as an internal standard, assuming that the response of the detector for all methyl fatty acids was identical.

\section{LIPID EMULSIONS}

Lipofundin 20\% (MCT/LCT; B. Braun Melsungen, Germany) is a $200 \mathrm{~g} / 1$ fat emulsion containing equal molar amounts of soybean oil LCT (100 g/l; mainly $\mathrm{C}_{16}, \mathrm{C}_{18: 1}$, and $\mathrm{C}_{18: 2}$ ), and MCT (100 g/l; mainly $\mathrm{C}_{8}$ and $\mathrm{C}_{10}$ ), emulsified in egg yolk lecithin (12 g/l), glycerol, and sterile water. Intralipid $20 \%$ (LCT, Pharmacia, Sweden) contains $200 \mathrm{~g} / 1$ of LCT soybean oil emulsified in egg yolk lecithin (12 g/1), glycerol, and sterile water.

STATISTICAL ANALYSIS

Data are presented as mean (SD). In order to compare mean values of bile and plasma lipid concentrations, as well as fatty acid compositions at the various time points, two way analysis of variance with repeated measures and Scheffe multiple comparison post hoc test, and simple contrast for repeated variables were performed.

\section{Results}

STUDY I: POSTOPERATIVE STABILISATION OF PLASMA AND BILIARY LIPIDS

In all patients, plasma cholesterol levels decreased following operation (fig 1). In patients with a bile fistula, there was a significant decrease (preoperation versus C-0, $\mathrm{p}<0.001)$; in the control group there was a similar trend $(\mathrm{p}=0.06)$. The relative decrease in plasma cholesterol level in patients with a bile fistula was $35 \%$ compared with $20 \%$ in the

Table 4 Effect of lipid on biliary lipid composition

\begin{tabular}{|c|c|c|c|c|c|c|c|c|c|}
\hline & \multicolumn{4}{|l|}{$L C T$} & \multicolumn{4}{|l|}{$M C T / L C T$} & \multirow[b]{2}{*}{ Control } \\
\hline & Time 0 & End (6h) & Post (9h) & Post $(24 h)$ & Time 0 & End (6h) & Post (9h) & Post (24h) & \\
\hline $\mathrm{CH}$ & $4.1(1.5)$ & $4.8(2.4)^{\star}$ & $3.0(0.9)$ & $3.0(0.7)$ & $3.6(1.4)$ & $5.1(1.8)^{\star}$ & $4.0(1.6)$ & $3.5(1.4)$ & $4.2(0.6)$ \\
\hline PL & $8.8(3.5)$ & $10.5(5.2)$ & $5.7(3.2)$ & $5.6(2.7)$ & $9.0(4.3)$ & $9.8(5.5)$ & $7.3(5.4)$ & $7.8(4.9)$ & $7.0(0.9)$ \\
\hline BS & $28.9(10.8)$ & $21.1(12.2)$ & $22.6(10.9)$ & $19.2(6.7)$ & $37.8(16.8)$ & $25.1(16.9)$ & $23.6(15.3)$ & $31.2(11.8)$ & $28.0(3.4)$ \\
\hline CH:PL & $0.5(0.2)$ & $0.6(0.2)$ & $0.5(0.2)$ & $0.6(0.3)$ & $0.4(0.1)$ & $0.5(0.1)$ & $0.6(0.2)$ & $0.5(0.2)$ & $0.6(0.1)$ \\
\hline Total lipids & $23 \quad(8)$ & 19 (6)† & 19 (11) & $15 \quad(5)$ & $26 \quad(12)$ & $16 \quad(10) \dagger$ & $9 \quad(12)$ & $22 \quad(10)$ & $21 \quad(1)$ \\
\hline CSI & $2.5(2.0)$ & $3.4(1.5)$ & $3.2(1.8)$ & $2.6(1.1)$ & $1.8(0.6)$ & $3.9(1.8)^{\star \star}$ & $2.9(1.4)$ & $1.3(0.8)$ & $2.4(0.4)$ \\
\hline Bile flow & $0.5(0.2)$ & $0.5(0.2)$ & $0.4(0.2)$ & $0.4(0.1)$ & $0.5(0.2)$ & $0.4(0.0)$ & $0.4(0.1)$ & $0.3(0.1)$ & $0.4(0.1)$ \\
\hline
\end{tabular}

Results are expressed as mean (SD). Control values represent means of three time points.

${ }^{\star} \mathrm{p}<0.05,{ }^{\star \star}{ }^{\star} \mathrm{p}<0.01,+\mathrm{p}<0.005$ compared with time 0 .

$\mathrm{CH}$, total (free) cholesterol; PL, phospholipids; BS, bile salts; CSI, cholesterol saturation index.

$\mathrm{CH}, \mathrm{PL}$, and $\mathrm{BS}$ are expressed in $\mathrm{mM}$, total lipids in $\mathrm{g} / \mathrm{l}$, bile flow in $\mathrm{ml} / \mathrm{min}$. 
control group $(p<0.05)$. Restoration of the enterohepatic circulation by clamping of the bile fistula resulted in a gradual (yet insignificant) increase in plasma cholesterol during the study period (from C-0 to C-6). By day 6 after clamping plasma cholesterol in patients with a bile fistula had reached a level equal to that of control patients.

On the day of clamping the relative decrease was significantly larger in fistula patients than controls $(\mathrm{p}<0.05)$. At six months plasma cholesterol level reached preoperative levels.

After six months, plasma cholesterol levels had returned in both groups to preoperative levels. The increase in cholesterol was statistically significant $(p<0.01$ relative to the first postoperative measurement, C-0) and was due to an increase in both free and esterified cholesterol. The concentrations of plasma TG and PL remained constant throughout the study period and were not affected by surgery or $\mathrm{T}$ tube clamping (data not shown).

Table 2 shows the biliary lipid concentrations of patients with a $\mathrm{T}$ tube. On clamping day (C-0), following continuous drainage of bile from the day of surgery, all biliary lipid concentrations were low (table 2). Clamping of the bile fistula resulted in significant increases in BS $(p<0.001), P L(p<0.05)$, and cholesterol concentrations $(p<0.05)$. The increased level of biliary cholesterol correlated with the increase observed in plasma cholesterol $(r=0.65$; $\mathrm{p}<0.05)$. BS, PL, and total lipid concentrations reached a plateau value after two days of clamping, while the stabilisation of cholesterol occurred somewhat later, by day 3 (table 2). Concomitantly with the above changes, the CSI decreased during the initial two days $(p<0.05)$. The cholesterol:PL ratio in bile decreased gradually, whereas bile flow increased insignificantly during the first four days after clamping (table 2).

After assessing the optimal conditions for studying the hepatic bile composition, we investigated the effects of lipid infusion on plasma and hepatic bile lipids.

STUDY II: EFFECTS OF LIPID INFUSION

Infusion of LCT as well as MCT/LCT lipid emulsions resulted in a significant increase in TG and PL concentrations in plasma (table 3). All plasma parameters returned to preinfusion levels after 24 hours (data not shown).

Infusion of both LCT and MCT/LCT affected hepatic bile lipid composition, but the effects of $\mathrm{MCT} / \mathrm{LCT}$ were more pronounced (table 4). LCT and MCT/LCT infusions

Table 5 Fatty acid composition of biliary phospholipids (weight\%)

\begin{tabular}{|c|c|c|c|c|c|c|}
\hline & \multicolumn{3}{|l|}{$L C T$} & \multicolumn{3}{|l|}{$M C T / L C T$} \\
\hline & Time 0 & End (6h) & Post $(9 h)$ & Time 0 & End $(6 h)$ & Post (9h) \\
\hline $16: 0$ & $38.0(1.1)$ & 37.2( & $36.9(0.9)^{\star}$ & $0.2(2.9)$ & $35.0(1.6)$ & $33.6(2.0)$ \\
\hline $18: 0$ & $4.8(0.6)$ & $4.9(0.5)$ & $4.9(0.6)$ & $5.6(0.4)$ & $5.4(0.3)$ & $5.5(0.4)$ \\
\hline $18: 1$ & $8.6(0.6)$ & $8.6(1.8)$ & $9.0(1.9)$ & $9.5(1.8)$ & $9.3(0.7)$ & $9.8(0.2)$ \\
\hline $18: 2$ & $30.8(3.5)$ & $37.7(1.1) \dagger$ & $37.5(1.5) \dagger$ & $32.0(2.9)$ & $35.8(1.2)^{\star}$ & $35.6(1.9)^{\star}$ \\
\hline $20: 4$ & $8.1(1.6)$ & $7.1(0.9)$ & $6.9(1.4)$ & $8.2(1.4)$ & $7.2(0.9)$ & $7.0(1.3)$ \\
\hline $22: 6$ & $2.2(0.8)$ & $1.7(0.3)$ & $1.7(0.4)$ & $2.5(0.5)$ & $2.1(0.1)$ & $2.1(0.1)$ \\
\hline
\end{tabular}

Results are expressed as mean (SD). Fatty acids comprising less than $1 \%$ of total acyl fatty acids are not included in this table.

${ }^{\star} \mathrm{p}<0.05,+\mathrm{p}<0.01$ compared with time 0 . caused a significant increase in biliary cholesterol concentration $(p<0.05)$, while PL did not change significantly. The cholesterol:PL ratio and CSI in the MCT/LCT group increased significantly $(p<0.05)$, while the respective changes after LCT infusion were not statistically significant. Total lipid concentration decreased significantly $(p<0.05)$ in both LCT and MCT/LCT infusions, but the decrease was more pronounced in the MCT/LCT group. The decrease in BS concentration was not significant. Bile flow remained unchanged throughout the study. In all groups, the parameters returned to preinfusion values after 24 hours. No changes were observed in hepatic bile lipid concentration of control patients infused with glucose saline only (table 4).

Both lipid infusions affected the fatty acyl composition of biliary PL as can be seen in table 5. A significant enrichment with linoleic acid $(18: 2)$ was seen after LCT $(p<0.01)$ as well as MCT/LCT $(p<0.05)$ infusions. This increase was accompanied by a decrease in palmitic (16:0) (significant for LCT infusion post (nine hours), $\mathrm{p}<0.05)$, arachidonic $(20: 4)$, and docosahexaenoic $(22: 6)$ fatty acids.

\section{Discussion}

In the present paper, we have studied the effects of an intravenous lipid infusion on plasma and hepatic biliary lipids in cholesterol gallstone patients after biliary surgery. Infusion of lipid emulsions containing MCT caused an increase in hepatic biliary cholesterol concentration, bile lithogenicity, and altered the fatty acid profile of biliary phospholipids. These changes were accompanied by increases in plasma triglycerides and phospholipids.

Biliary lipid concentrations were measured in bile collected through a $\mathrm{T}$ tube. As drainage from a bile fistula may disturb the enterohepatic circulation, it was desirable to perform the study under conditions in which changes in bile lipids due to interrupted enterohepatic circulation would not seriously interfere with the changes induced by the lipid infusions. Therefore, we first defined the study conditions in a control group of patients who did not receive lipid infusion.

The results of the first study show that continuous drainage of bile through a $\mathrm{T}$ tube after cholecystectomy and choledochotomy notably decreases biliary lipids and to a lesser extent plasma cholesterol levels. Restoration of the enterohepatic circulation by clamping of the $T$ tube resulted in an increase in both plasma and biliary lipid concentrations with a slight, insignificant increase in bile flow rate. Biliary lipids reached plateau values within three days. Similar results were reported by Shaffer $e t a l^{16}$ who found that clamping of the $\mathrm{T}$ tube resulted in restoration of the enterohepatic circulation within two to three days. Plasma cholesterol levels took longer to recover, but reached levels equal to control patients within six days after clamping. As bile flow is strongly associated with bile salt secretion, one might also expect an increase in the bile flow after reestablishment of the enterohepatic circulation. It has to be remembered, however, that the bile collec- 
tion period was only 30 minutes in our study and the results are expressed as $\mathrm{ml}$ per one minute. The bile flow rate varies during 24 hours and the calculation per one minute tends to minimise actual changes and might not accurately reflect the daily bile flow. This might explain the very moderate increase in bile flow found in our six patients.

It should be noted that the effects observed in the present study are due to a combination of $\mathrm{T}$ tube drainage and cholecystectomy. Cholecystectomy has been shown to have effects similar to those observed in the combined situation, but to a significantly lesser extent. ${ }^{27-29}$ The present study shows that the specific effects of bile fistula on plasma and bile lipids are reversed in less than six days after $\mathrm{T}$ tube clamping. Therefore, studies examining the effects of drugs, nutrients, or other substrates on bile should not be performed on bile collected from a continuously draining $\mathrm{T}$ tube, but on bile collected after clamping for a period of three to six days or longer. ${ }^{30} 31$

Based on these conclusions we studied the effects of short term lipid infusion on plasma and hepatic biliary lipids in cholesterol gallstone patients (study II) at least five days after $\mathrm{T}$ tube clamping, using each patient as his or her own control.

In plasma, similar to previous reports, ${ }^{32}{ }^{33}$ the lipid infusion resulted in a significant but short term increase in the levels of TG and PL.

Less is known about the effects of lipid infusion on biliary lipid composition in humans. We have previously shown that lipid infusion, and MCT/LCT in particular, increases biliary cholesterol and total lipid concentration with a resultant increase in gall bladder bile lithogenicity in non-gallstone patients. ${ }^{9}$ In the present study a significant rise in hepatic biliary cholesterol was shown following the administration of both lipid emulsions, but more with intravenous MCT/LCT lipid emulsion. More specifically, infusion of both lipid emulsions reduced the BS concentration and increased the cholesterol concentration in the bile $(42 \%$ for MCT/LCT and $20 \%$ for LCT). These results reinforce our previous report ${ }^{9}$ and support our hypothesis that MCT/LCT infusion increases gall bladder bile lithogenicity by directly affecting hepatic bile composition and not only through gall bladder bile stasis. The assumption that the above effects induced by infusion of lipid emulsions are not variations due to circadian rhythm of hepatic bile composition are supported by the lack of any effect observed by glucose saline infusion in control patients.

The time for hepatic uptake, degradation of MCT, and its conversion to biliary cholesterol has not been measured in man. However, based on studies with radiolabelled tracers done in patients with a bile fistula with related compounds, it has been estimated that the labelled product appears in the bile within 1.5-10 hours postadministration. ${ }^{31}$ Our data are based on bile collected at the end of six hours of constant infusion, which is within this time frame.
Previously, we have reported that infusion of MCT/LCT failed to show an effect on gall bladder bile composition in cholesterol gallstone patients. ${ }^{34}$ This is in contrast to nongallstone patients in whom the biliary cholesterol rose to a significantly higher level. ${ }^{9}$ It is likely that in cholesterol gallstone patients the lack of change in gall bladder bile composition might be due to precipitation of the MCT/ LCT induced excess cholesterol within the gall bladder. The gall bladder bile of these patients is known to be saturated by cholesterol and to contain an excess of pronucleating and/or be deficient in antinucleating factors. This hypothesis is in agreement with the results of the present study, in which we could show the lithogenic effects of MCT/LCT infusion on the composition of hepatic bile in cholesterol gallstone patients. Moreover, the above hypothesis is supported by the recent in vitro observation of van den Berg et $a l^{55}$ who have shown that cholesterol gallstones act as a cholesterol sink for precipitation of cholesterol from saturated bile.

The mechanism by which fat emulsion containing MCT increases biliary cholesterol levels is unknown. It seems, however, to be related to the different metabolic pathways of medium chain fatty acids compared with long chain fatty acids within the liver. ${ }^{36}$ Beta oxidation of medium chain fatty acids to acetyl CoA units results in a surplus of building blocks for lipid synthesis, especially that of cholesterol. This suggestion of enhanced de novo synthesis in the liver of biliary lipids is supported by the finding that the fatty acid profile of bile phospholipids was altered by lipid infusion. The proportion of the essential fatty acid linoleic acid (18:2) increased significantly in hepatic biliary PL after infusion of both lipid emulsions. The rise was more pronounced after LCT infusion, as the amount of 18:2 in LCT is twofold higher than in the MCT/LCT emulsion and this fatty acid is not synthesised in the body. Similar results were obtained after feeding a diet enriched with phosphatidylethanolamine containing a high proportion of linoleic acid. ${ }^{37}$

In conclusion, these results show that short term infusion of lipid emulsions containing LCT and MCT notably affects biliary lipid composition, fatty acid profile, and bile lithogenicity. This effect, shown especially by MCT, can contribute independently to biliary sludge formation by directly altering bile composition. The lithogenic effect of long term infusion of this emulsion, coupled with gall bladder stasis, may lead to a greater risk of gallstone formation. This work was supported in part by the Schreiber Fund and
Minerva Centre for Cholesterol Gallstones and Lipid MetaboMinerva Centre for Cholesterol Gallstones and Lipid Metabo-
lism in the Liver, Tel Aviv University. The work was performed in partial fulfilment of the requirements for a $\mathrm{PhD}$ degree of $\mathrm{R}$ in partial fulfilment of the requirements for a PhD degree of $\mathrm{R}$
Pakula, Sackler Faculty of Medicine, Tel Aviv University, Israel. Pakula, Sackler Faculty of Medicine, Tel Aviv University, Israel.
The authors wish to thank Professor Dov Lichtenberg from the Department of Pharmacology and Physiology, Tel-Aviv University, for his assistance in the preparation of this manuscript.

1 Meesing B, Bories C, Kunstlinger F, et al. Does total parenteral nutrition induce gallbladder sludge formation and lithiasis? Gastroenterology 1983;84:1012-19. 
2 Roslyn JJ, Pitt HA, Mann LL, et al. Gallbladder disease in patients on long-term parenteral nutrition. Gastroenterology

3 Holzbach T. Gallbladder stasis: consequence of long-term parenteral hyperalimentation and risk factor for cholelithiasis. Gastroenterology 1983;84:1055-8

4 Hughes CA, Talbot IC, Ducker DA, et al. Total parenteral nutrition in infancy: effect on the liver and suggested pathogenesis. Gut 1983;24:241-8.

5 Sitzmann JV, Pitt HA, Steinborn PA, et al. Cholecystokinin prevents parenteral nutrition induced biliary sludge in humans. Surg Gynecol Obstet 1990;170:25-31.

6 Doty J, Pitt H, Porter-Fink V, et al. Cholecystokinin prophylaxis of parenteral nutrition-induced gallbladder disease. Ann Surg 1985;201:76-80.

7 Rubin M, Halpern Z, Livoff A, et al. The effect of lipid infusion on liver function and biliary secretion in rats. Lipids 1992;27:321-5.

8 Gimmon Z, Kelley RF, Simko V, et al. Total parenteral nutrition increases lithogenicity in rat. $\mathcal{F}$ Surg Res $1982 ; 32$. 256-63.

9 Rubin M, Halpern Z, Charach G, et al. Effect of lipid infusion on bile composition and lithogenicity in patients withsion on bile composition and lithogenicity in patien

10 Booker ML, LaMorte WW, Beer ER, et al. Effects of dietary cholesterol and triglycerides on lipid concentrations in liver, plasma, and bile. Lipids 1997;32:163-72

11 Stahlberg D, Reihner E, Angelin B, et al. Interruption of the enterohepatic circulation of bile acids stimulates the esterification rate of cholesterol in human liver. F Lipid Res 1991; 32:1409-15.

12 Farkkila MA, Telvis RS, Miettinen TA. Cholesterol absorption regulates cholesterol metabolism and plasma lipoprotein level in patients with gut exclusions. Gastroenterology 1988;94:582-9.

13 Akerlund J, Reihner E, Angelin B, et al. Hepatic metabolism of cholesterol in Crohn's disease: effect of partial resection of ileum. Gastroenterology 1991;100:1046-53.

14 Reihner E, Angelin B, Rudling M, et al. Regulation of hepatic cholesterol metabolism in humans: stimulatory hepatic cholesterol metabolism in humans: stimulatory effect of cholestyramine on $\mathrm{HMG}$-CoA reductase activity and low density lipoprotein receptor exp

15 Angelin B, Leijd B. Hultcrantz R, et al. Increased turnover of very low density lipoprotein triglyceride during treatment with cholesteramine in familiar hypercholesterolaemia. $\mathcal{F}$ Intern Med 1990;227:201-6.

16 Shaffer EA, Braasch JW, Small DM. Bile composition at and after surgery in normal persons and patients with gallstones. N Engl f Med 1972;287:1317-22.

17 Peled Y, Halpern Z, Baruch R, et al. Cholesterol nucleation from its carriers in human bile. Hepatology 1988;8:914-18.

18 Folch J, Lees M, Sloane Stanley GH. A simple method for the isolation and purification of total lipids from animal tissues. F Biol Chem 1957;226:497-509.

19 Talalay P. Enzymatic analysis of steroid hormones. Biochem Anal 1960;8:119-43.

20 Abbell LL, Levy BB, Brodie BB, et al. A simplified method for the estimation of total cholesterol in serum and demonstration of its specificity. F Biol Chem 1952;195:357-66.
21 Bartlett G. Phosphorus assay in column chromatography. Biol Chem 1958;234:466-8.

22 Carey MC. Critical tables for calculating the cholesterol saturation of native bile. $\mathcal{F}$ Lipid Res 1978;19:945-56.

23 van Erpecum KJ, van Berge Henegouwen GP, Stoelwinder $\mathrm{B}$, et al. Cholesterol and pigment gallstone disease: comparison of the reliability of three bile tests for differentiation between the two stone types. Scand 7 Gastroenterol 1988;23:948-54.

24 Havel RJ, Eder HA, Bragdon JH. The distribution and chemical composition of ultracentrifugally separated lipoproteins in human serum. $\mathcal{F}$ Clin Invest 1955;34:1345-53.

25 Christie WW. The preparation of derivatives of lipids. In: Lipid analysis. 2nd edn. London: Pergamon Press, 1982: $51-61$.

26 Schlenk H, Gellerman JL. Esterification of fatty acids with gas methane on a small scale. Anal Chem 1960;32:1412-

27 Kuliak-Ublick GA, Paumgartner G, Berr F. Long-term effects of cholecystectomy on bile acid metabolism. Hepatology 1995;21:41-5.

28 Berr F, Stellaard F, Pratschke E, et al. Effects of cholecystectomy on the kinetics of primary and secondary bile acids. $f$ Clin Invest 1989;83:1541-50.

29 Juvonen T, Kervinen K, Kairaluoma MI, et al. Effect of cholecystectomy on plasma lipid and lipoprotein levels. Hepatogastroenterology 1995;42:377-82.

30 Schwartz CC, Zech LA, Vanden Vroek JM, et al. Cholesterol kinetics in subjects with bile fistula, positive relationship between size of the bile acid precursor pool and bile acid synthetic rate. f Clin Invest 1993;91:923-38.

31 Murarca M, Baggio G, Miconi L, et al. Acute effects of HMG-CoA reductase inhibitors on biliary lipids with interrupted enterohepatic circulation. Eur 7 Clin Invest 1991;21:204-8.

32 Carpentier YA. Intravascular metabolism of fat emulsions. Clin Nutr 1989;8:115-25.

33 Granot E, Deckelbaum RJ, Eisenberg S, et al. Core modification of human low-density lipoprotein by artificial triacylglycerol emulsion. Biochim Biophys Acta 1985;833: 308-15.

34 Rubin M, Pakula R, Moser A, et al. Does lipid infusion affect bile composition in humans? Isr F Med Sci 1996;32:130813.

35 van den Berg AA, van Buul JD, Tytgat GNJ, et al. Cholesterol gallstone growth in model bile is inversely related to cholesterol crystal formation [abstract]. Gastroenterology 1998;114:A547.

36 Pakula R, Rubin M, Moser AM, et al. Biosynthesis of medium chain triacylglycerols and phospholipids by HepG-2 cells. Lipids 1997;32:489-95

37 Pakula R, Konikoff FM, Rubin M, et al. The effect of dietary phospholipids enriched with phosphatidylethanolamine on bile and red cell membrane lipids in humans. Lipids 1996; 31:295-303. 